\title{
Poly(thioether) Vitrimers via Transalkylation of Trialkylsulfonium Salts
}

\author{
Benjamin Hendriks, Jelle Waelkens, Johan M. Winne,* and Filip E. Du Prez*0
}

Department of Organic and Macromolecular Chemistry, Polymer Chemistry Research Group and Laboratory of Organic Synthesis, Ghent University, Krijgslaan, 281, S4-bis, B-9000 Ghent, Belgium

\section{Supporting Information}

ABSTRACT: Vitrimers are permanently cross-linked organic polymers that can be reshaped, molded, and recycled without loss of network integrity. Herein, we report poly(thioether) networks, prepared through a straightforward thiol-ene photopolymerization, that can be turned into catalyst-free vitrimer materials by partial alkylation of the thioethers (1$10 \%)$ to the corresponding trialkylsulfonium salts. Based on a classical $\mathrm{S}_{\mathrm{N}}$ 2-type substitution, the resulting polyionic networks can be reshaped upon heating via swift transalkylation reactions. This novel exchange reaction for the design of vitrimers was studied both on low MW model compounds as well as on a material level. In addition, we demonstrated the recycling of these networks without significant loss of mechanical properties.

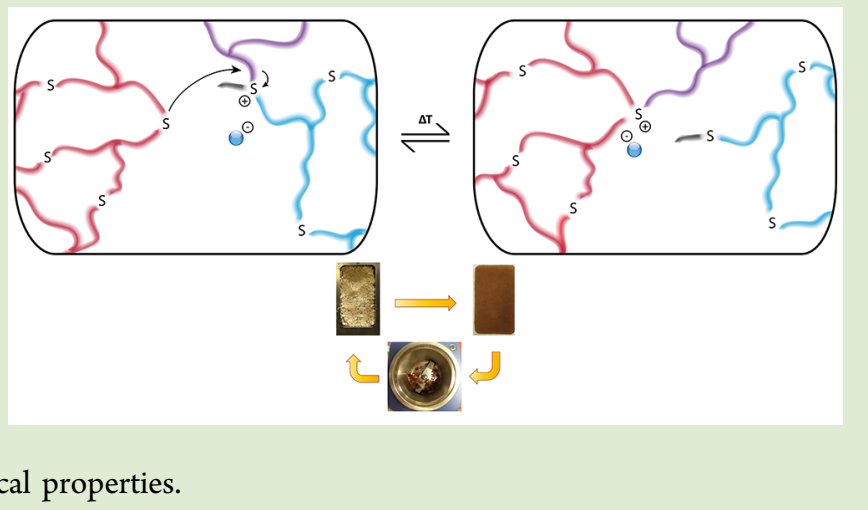

$\mathrm{T}$ he desirable but antipodal characteristics of thermosetting and thermoplastic polymers, respectively, resistance to deformation or dissolution, and thermal plasticity for processing and recycling can be achieved by the introduction of exchangeable chemical bonds in a polymer network. Networks containing exchangeable cross-links are also known as covalent adaptable networks (CANs). ${ }^{1}$ A subset of CANs rely on an associative (or concerted) bond exchange reaction, rather than the more common dissociative exchange reactions in which bonds are first broken and then reformed. ${ }^{2}$ Uniquely, associative bond exchanges permit network reorganizations and stress relaxation while maintaining a constant cross-linking density. At higher temperature, the viscosity of such materials is solely controlled by the rate of the chemical exchange reactions. Thermally triggered associative CANs have been classified as vitrimers by Leibler and co-workers and combine excellent mechanical properties at service temperatures and malleability and reprocessing by heating without precise control of temperature. ${ }^{3}$

Vitrimer materials, or vitrimer behavior of organic polymers, were first discovered by Leibler and co-workers, in polyesters that can undergo transesterification exchange by addition of a suitable catalyst. ${ }^{4}$ Next to Leibler's initial vitrimers (Figure 1A), a limited but growing number of other exchange reactions have been explored and found to give vitrimer-like properties to polymers. ${ }^{5}$ Examples of other suitable chemistries include exchange reactions of vinylogous urethane bonds, ${ }^{6}$ carbamate bonds, ${ }^{9}$ siloxane bonds, ${ }^{8}$ disulfide bonds, ${ }^{9}$ imine bonds, ${ }^{10}$ olefin bonds, ${ }^{11}$ dioxaborolane ester bonds, ${ }^{12}$ and 1,2,3-triazolium alkyl bonds. ${ }^{13}$ The latter exchange reaction is the first reported transalkylation reaction to be used as vitrimer materials (Figure 1B), which also shows potential for applications in solid

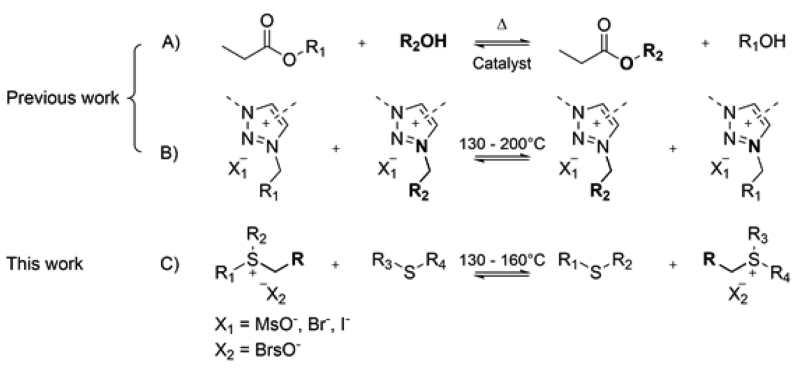

Figure 1. Schematic overview of transesterification ${ }^{3}$ (A), C-N transalkylation $^{13}$ (B), and transalkylation of sulfonium salts (C) used for the design of vitrimer materials.

electrolytes, due to the ionic nature of the cross-links. ${ }^{14}$ In this work, we have explored an alternative chemistry platform for transalkylating vitrimer materials, based on a classical $S_{N} 2$-type reaction of sulfonium salts with thioether nucleophiles (Figure $1 C)$. In fact, the possibility of an exchange reaction between sulfides (thioethers) and sulfonium salts has been previously explored as a reversible propagation step in the cationic ringopening polymerization of cyclic thioethers, in seminal work by Goethals et al. ${ }^{15}$ From these and other studies, benzylic sulfonium salts and thioethers are known to readily exchange alkyl chains at room temperature without requiring any additives or catalysts. At the outset of the work reported herein, we wanted to investigate the potential of this exchange reaction in catalyst-free polythioether-based vitrimers. For this,

Received: July 7, 2017

Accepted: August 10, 2017

Published: August 15, 2017 
we envisaged the hereunto little-known transalkylation of less activated aliphatic sulfonium salts and thioethers. Poly(thioether) networks are readily prepared by the photoinduced thiol-ene reaction of alkene and thiol monomers in bulk. ${ }^{16}$ Exchangeable trialkylsulfonium bonds should be easily introduced by partial alkylation of the thioether functions, resulting in poly(thioether-sulfonium salt) networks (PTESSs). Indeed, we have found PTESS materials that are insoluble and show short relaxation times at elevated temperatures. We also report the simple recyclability of PTESSs by grinding and remolding them repeatedly, whereas nonalkylated polythioethers cannot undergo this recycling and thermoforming process.

To evaluate the exchange kinetics of simple aliphatic trialkylsulfonium salts, a model study on low MW compounds was performed. First, model compound 1 (Figure 2a) was

a)

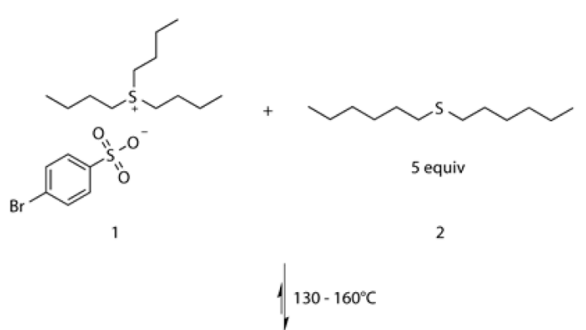

b)
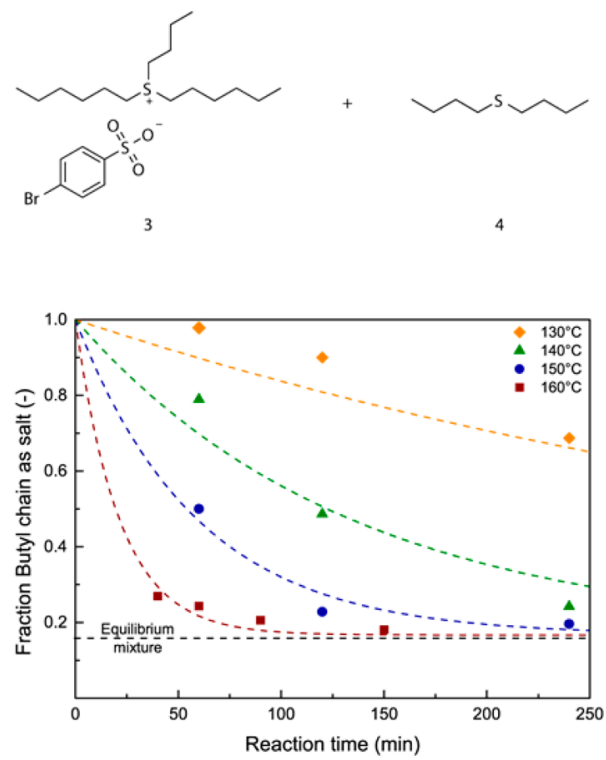

Figure 2. (a) Exchange reaction on low MW compounds for a kinetic study. For convenience, the butyl hexyl sulfide and hexyl dibutyl sulfonium salt as exchange products were not drawn. (b) Disappearance of the butyl side chain as a salt of compound $\mathbf{1}$ by exchange reactions as a function of time for different temperatures $\left(130-160{ }^{\circ} \mathrm{C}\right)$.

prepared by the alkylation of butyl sulfide with butyl $p$ bromobenzenesulfonate (brosylate). Brosylates are stable, nonnucleophilic counterions, and alkylbrosylates also proved to be the most promising alkylating reagents in a small screening process. In each exchange experiment, the tributylsulfonium salt $\mathbf{1}$ is treated with 5 equiv of dihexyl sulfide $\mathbf{2}$ without solvent, and the resulting mixtures were heated at 130,140,150, and $160{ }^{\circ} \mathrm{C}$. NMR spectra were recorded at different time intervals. ${ }^{1} \mathrm{H}$ NMR was not suitable for a quantitative study of the exchange kinetics due to spectral overlap, but the trans- alkylation reaction could be easily monitored using ${ }^{13} \mathrm{C}$ NMR (Figure S1, Supporting Information). From the NMR integration values, the remaining fraction of a butyl group in the sulfonium salt is plotted versus the reaction time (Figure 2b). Notwithstanding the limited accuracy of ${ }^{13} \mathrm{C}$ NMR integration, approximative reaction half-lives can be determined at each temperature, ranging from a few hours (at $130{ }^{\circ} \mathrm{C}$ ) to just a few minutes (at $160{ }^{\circ} \mathrm{C}$ ). From the fitted curves, an activation energy of $108 \pm 4 \mathrm{~kJ} \mathrm{~mol}^{-1}$ was calculated for the exchange reaction (Figure S2, Supporting Information). This activation energy is around $30 \mathrm{~kJ} \mathrm{~mol}^{-1}$ lower compared to the transalkylation of 1,2,3-triazolium bromide salts, in line with the higher nucleophilicity in an $\mathrm{S}_{\mathrm{N}} 2$ reaction expected for a thioether compared to a triazole. ${ }^{13}$

With the possible exchange of alkyl groups between trialkyl sulfonium salts and thioethers established in our model study, we then set out to implement this chemistry in poly(thioether) networks. However, cross-linking of linear poly(thioether) chains with bifunctional alkylating agents proved to be a hard to control process in bulk, due to solubility and phase separation issues. Working with solvents, it proved hard to reach high cross-linking conversions. Finally, an alternative strategy proved much more successful, in which cross-linked materials are directly prepared in a thiol-ene photopolymerization using multifunctional monomers $(f>2)$. Exchangeable bonds were readily introduced into these static networks by treatment with an alkylating agent. We initially explored alkylation of solventswollen networks, but as the alkylation reaction requires significant heating for its activation, we found that optimal results are obtained when alkyl brosylates are first physically incorporated into the networks by simply adding them to the monomer mixture before the photo-cross-linking polymerization is performed at low temperature. A thermal cure then covalently incorporates this additive through alkylation of the network thioether functions. Following a brief screening of possible network building blocks, the completely aliphatic octanedithiol 5, octadiene 6 , and the more rigid trithiol ${ }^{17} 7$ were identified as suitable and readily available generic aliphatic monomers. The butyl brosylate $\mathbf{8}$ was chosen as a simple alkylating agent (Figure 3). All compounds (monomers 5, 6,

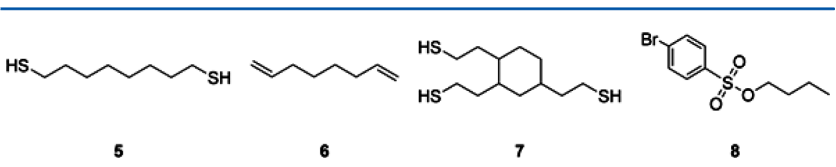

Figure 3. Monomers used for production of vitrimer materials. A small excess of diene ( 1.02 equiv) was used to limit the number of free thiols after polymerization. Compound $\mathbf{8}$, butyl brosylate, was used as alkylating agent.

and 7 , the initiator (DMPA), and the alkylating agent 8) were mixed, giving a homogeneous liquid mixture that was then injected between two glass plates separated by a spacer. The thiol-ene radical polymerization is initiated by UV irradiation $(365 \mathrm{~nm})$. After $1 \mathrm{~h}$ of UV irradiation, the samples were submitted to a thermal cure of $90 \mathrm{~min}$ at $140{ }^{\circ} \mathrm{C}$, giving defectfree samples that should have undergone complete alkylation, as expected from the low MW model alkylation reactions (see Figure S3, Supporting Information). The conversion of butyl brosylate in the polymer matrix was also verified using FT-IR analysis of the materials showing the complete disappearance of the diagnostic carbon-sulfonate stretch band within $1 \mathrm{~h}$ of heating at $140{ }^{\circ} \mathrm{C}$ (Figure S4, Supporting Information). 
As a control experiment to check for "premature" alkylation reactions during the exothermic photo-cross-linking polymerization, a thiol and butyl brosylate were incubated at different temperatures (up to $70{ }^{\circ} \mathrm{C}$ ). Even after $4 \mathrm{~h}$ at $70{ }^{\circ} \mathrm{C}$, corresponding to a quite harsh photopolymerization procedure, both thiol and brosylate functions remain completely unchanged as judged by ${ }^{1} \mathrm{H}$ NMR.

Using the above-described protocol, PTESS networks were prepared with different stoichiometries of the various low MW components $5,6,7$, and 8 . In the absence of alkylating agent 8 , no significant stress relaxation was observed at elevated temperatures, as can be expected. As the number of introduced exchangeable bonds (two per sulfonium salt moiety) is increased, a faster network reorganization should be possible. Indeed, we not only found stress relaxation in these networks but also observed that increasing the degree of alkylation leads to a significant decrease in relaxation times: going from 1 to 10 mol \% butyl brosylate, relaxation times drop from more than 75 min to only $10 \mathrm{~min}$ at $150{ }^{\circ} \mathrm{C}$ (Figure 4a). The networks are thus effectively able to relax stress with relaxation times closely mimicking the observed "chemical" relaxation times in our model system (Figure 2b). We additionally found that the degree of alkylation also has an effect on the swelling ratio and soluble fractions of the synthesized networks (see SI: Table 1). The degree of alkylation has a rather pronounced effect on the soluble fraction (going up from $6 \%$ to $15 \%$ going from $1 \%$ to $10 \%$ alkylation degree). An increase in soluble fraction is likely caused here by network chain ends that are dynamically cleaved off by exchange to a butyl group, thus dislodging themselves from the bulk material, rather than by intrinsic network deficiencies or degradation. Indeed, incorporation of the butyl group as a thioether chain end in the soluble fraction can be observed from ${ }^{1} \mathrm{H}$ NMR of the Soxhlet residues (see Figure S5, Supporting Information). We also varied the ratio of thiol and alkene moieties in the PTESS networks. A near 1:1 stoichiometry is normally needed to reach high conversions in the photopolymerization. Remaining unreacted free thiols can be alkylated by the added $\mathbf{8}$ during the thermal cure, which would result in a net loss of exchangeable bonds. To avoid this obvious issue, we decided to always use a small excess of alkene (1.02:1). Within these boundaries, the ratio of dithiol 5 and trithiol 7 can still be varied almost at will. A 3:2 ratio of these monomers was found to be a good compromise giving insoluble networks that are not too brittle.

The polyionic PTESS networks obtained above with varying amounts of alkylating agent 8 , and varying ratios of 5 and 7 , were all rubbery networks at room temperature without a clearly observable $T_{\mathrm{g}}$ (Figure S6, Supporting Information). However, a high melting peak was observed between 30 and 60 ${ }^{\circ} \mathrm{C}$ with melt enthalpies up to $90 \mathrm{~J} / \mathrm{g}$, depending on the amount of trifunctional cross-linker 7 used (Figure S7, Supporting Information). Dynamic mechanical analysis confirmed the presence of a network with a rubbery plateau of around 1.1 $\mathrm{MPa}$ and showed a storage modulus of $80 \mathrm{MPa}$ for the networks containing a dithiol:trithiol molar ratio of 3:2 and 5 mol \% alkylating agent (see further Figure 5b). By thermogravimetric analysis (TGA), the PTESS networks proved to have good thermal stability with a mass loss of $5 \%$ at $250{ }^{\circ} \mathrm{C}$ (Figure S8, Supporting Information). An isothermal TGA experiment, performed at $160{ }^{\circ} \mathrm{C}$ to mimic reprocessing conditions (vide infra), showed negligible mass loss after $2 \mathrm{~h}$ (Figure S9, Supporting Information). a)

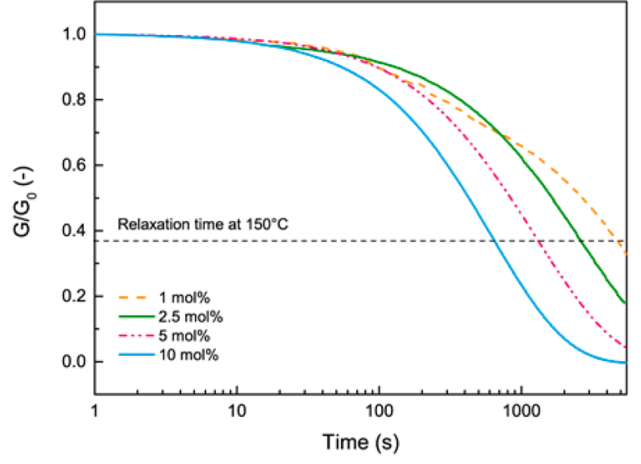

b)

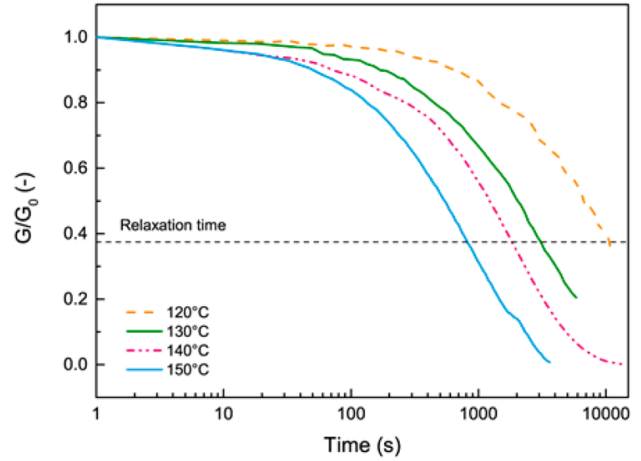

c)

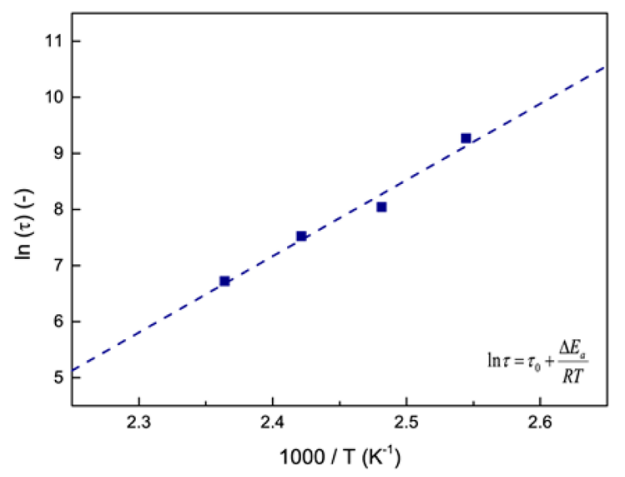

Figure 4. (a) Normalized stress-relaxation curves for networks (dithiol:trithiol 3:2) containing different alkylating agent loadings at $150{ }^{\circ} \mathrm{C}$. (b) Normalized stress-relaxation curves at different temperatures for networks comprising a dithiol:trithiol molar ratio of $3: 2$ and $5 \mathrm{~mol} \%$ alkylating agent. (c) Fitting of the relaxation times to the Arrhenius equation.

The vitrimer-like behavior of the PTESS networks was finally studied by extensive stress-relaxation experiments at different temperatures, to establish an Arrhenian relation between viscosity and temperature, the common diagnostic feature of vitrimers. ${ }^{18}$ All prepared networks, except the reference material without the alkylating additive $\mathbf{8}$, can completely relax the applied stress at elevated temperatures (Figure $4 \mathrm{~b}$ ), which is a good indicator of a swift exchange possible at all thioether linkages. (Figure S10, Supporting Information). Fitting the relaxation times to the Arrhenius equation gives a calculated activation energy of $113 \pm 12 \mathrm{~kJ} \mathrm{~mol}^{-1}$ from the slope (Figure 4c), in close agreement with the low MW chemical kinetics studies $(108 \pm 4 \mathrm{~kJ} / \mathrm{mol})$. In addition, the relaxation times allow for the calculation of the topology freezing temperature, $T_{\mathrm{v}}{ }^{3,19}$ This transition temperature is conventionally chosen as the temperature where the viscosity reaches $10^{12} \mathrm{~Pa} \mathrm{~s}^{20}$ The value of $T_{\mathrm{v}}$ can be extrapolated from 


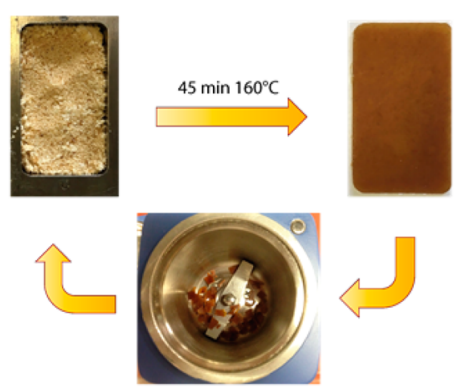

b)

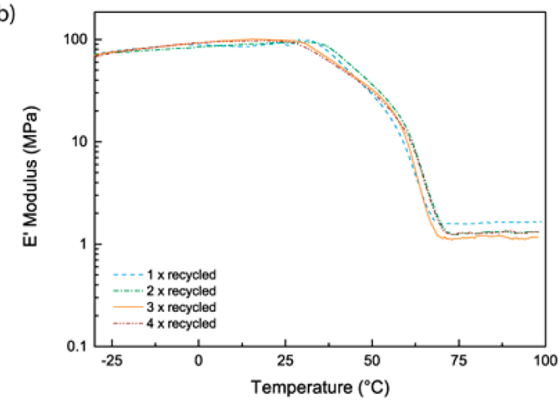

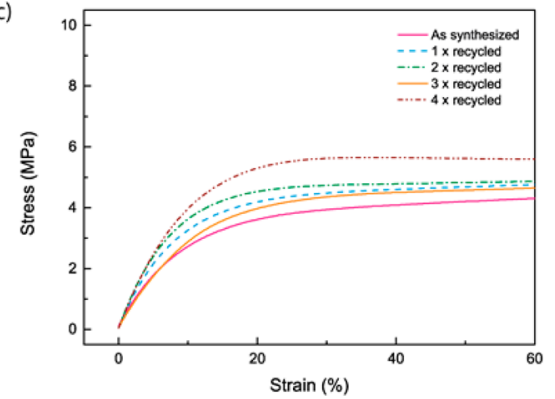

Figure 5. (a) Repeated recycling of vitrimer material by grinding and consecutive compression molding. (b) DMA curves of the recycled samples show recovery of the material properties. (c) Stress-strain curves of the recycled samples show recovery of the material properties.

the Arrhenius plot to a relaxation time of $2.8 \times 10^{6} \mathrm{~s}$ and results in a $T_{\mathrm{v}}$ of $65{ }^{\circ} \mathrm{C}$, which is well above the presumable $T_{\mathrm{g}}$ of the material in the range of $-20{ }^{\circ} \mathrm{C} .^{21}$ Thus, as long as the temperature is lower than this $T_{\mathrm{v}}$, the network topology is frozen, and the material behaves like a normal cross-linked elastomer.

To test the recyclable nature of the produced sulfonium salt networks, samples were first ground into small particles and then used as raw material for compression molding (Figure 5a). This grinding and molding process was repeated four times on the same sample. In addition, the grinded and remolded samples were subjected each time to differential scanning calorimetry (DSC), thermogravimetric and dynamic mechanical analysis (TGA \& DMA), tensile testing, solubility experiments, and FTIR. No significant change was observed in thermal stability ( $>5 \%$ mass loss), melting temperature (between 30 and $60{ }^{\circ} \mathrm{C}$ ), and enthalpy (Figures S8 and S11, Supporting Information). DMA revealed the full recovery of mechanical properties after recycling as the rubbery plateau was constant (Figure 5b). In addition, tensile tests showed the restoration of material properties (Figure 5c) with, however, a slight increase in young modulus and decrease in maximal stress compared to the virgin sample (Figure S12, Supporting Information). The increase in young modulus would suggest an increase in cross-linking density, following the first thermal recycling. However, this was not confirmed by swelling experiments, in which no change in swelling ratio was observed even after four recycling steps (Table S1, Supporting Information). Thus, the higher Young modulus can be attributed to the increased network density (packing) in recycled samples. Indeed, after grinding, the sample is put into a mold at $160{ }^{\circ} \mathrm{C}$ at a pressure of $1 \mathrm{ton} / \mathrm{m}^{2}$. Subsequent cooling fixes the more compact shape of the network by crystallization. The decrease in maximal stress likely originates from the loss of strain of all recycled samples compared to the virgin material (strain at break: as synthesized $250 \%$ - recycled $150 \%$ ). In further agreement with DMA, solubility experiments revealed no variation in soluble fraction, with the soluble fractions of the as-synthesized material and recycled samples being around $12 \%$ (Table S2, Supporting Information). Moreover, IR spectra before and after four consecutive recycling cycles are almost identical (Figure S13, Supporting Information) and show no sign of chemical degradation.

In summary, we have demonstrated that alkylated poly(thioether) networks, also referred to as poly(thioether)sulfonium salts (PTESSs), display vitrimeric behavior without requiring an embedded catalyst. In analogy with another catalyst-free system developed by us, being the transamination reactions with vinylogous acyl compounds, ${ }^{6}$ the PTESS materials show a markedly higher activation energy (113 vs $60 \mathrm{~kJ} \mathrm{~mol}^{-1}$ ). This results in a more pronounced temperature dependency that can be highly beneficial for processing purposes. The required alkylating agent is covalently incorporated in the network after polymerization by a thermal cure, and its quantity can be changed at will without affecting the network constitution. Here, semicrystalline vitrimers with a $T_{\mathrm{m}}$ ranging between 30 and $60{ }^{\circ} \mathrm{C}$ showed stress-relaxation times in the order of minutes at $160{ }^{\circ} \mathrm{C}$ without the use of any catalyst. These polyionic networks can be recycled and remolded without loss of mechanical properties. We expect PTESS vitrimers will be a powerful addition to the growing number of readily available polymer matrices that can benefit from vitrimer properties. In principle, this transalkylation chemistry should be transferable to all suitable sulfidecontaining materials.

\section{ASSOCIATED CONTENT}

\section{Supporting Information}

The Supporting Information is available free of charge on the ACS Publications website at DOI: 10.1021/acsmacrolett.7b00494.

Experimental details, material characterization, and supplemental figures and tables (PDF)

\section{AUTHOR INFORMATION}

\section{Corresponding Authors}

*E-mail: filip.duprez@ugent.be.

*E-mail: johan.winne@ugent.be.

ORCID $\odot$

Filip E. Du Prez: 0000-0003-4065-6373

Notes

The authors declare no competing financial interest.

\section{ACKNOWLEDGMENTS}

Wim Denissen is thanked for his assistance and guidance in this research. B.H. acknowledges Ghent University for financial support.

\section{DEDICATION}

Dedicated to the 80th anniversary of Prof. em. Eric Goethals.

\section{REFERENCES}

(1) (a) Kloxin, C. J.; Scott, T. F.; Adzima, B. J.; Bowman, C. N. Covalent Adaptable Networks (CANs): A Unique Paradigm in CrossLinked Polymers. Macromolecules 2010, 43 (6), 2643-2653. (b) Bowman, C. N.; Kloxin, C. J. Covalent Adaptable Networks: Reversible Bond Structures Incorporated in Polymer Networks. Angew. Chem., 
Int. Ed. 2012, 51 (18), 4272-4274. (c) Kloxin, C. J.; Bowman, C. N. Covalent adaptable networks: smart, reconfigurable and responsive network systems. Chem. Soc. Rev. 2013, 42 (17), 7161-7173.

(2) Billiet, S.; De Bruycker, K.; Driessen, F.; Goossens, H.; Van Speybroeck, V.; Winne, J. M.; Du Prez, F. E. Triazolinediones enable ultrafast and reversible click chemistry for the design of dynamic polymer systems. Nat. Chem. 2014, 6 (9), 815-821.

(3) (a) Montarnal, D.; Capelot, M.; Tournilhac, F.; Leibler, L. SilicaLike Malleable Materials from Permanent Organic Networks. Science 2011, 334 (6058), 965-968. (b) Capelot, M.; Unterlass, M. M.; Tournilhac, F.; Leibler, L. Catalytic Control of the Vitrimer Glass Transition. ACS Macro Lett. 2012, 1 (7), 789-792.

(4) (a) Altuna, F. I.; Pettarin, V.; Williams, R. J. J. Self-healable polymer networks based on the cross-linking of epoxidised soybean oil by an aqueous citric acid solution. Green Chem. 2013, 15 (12), 33603366. (b) Yu, K.; Taynton, P.; Zhang, W.; Dunn, M. L.; Qi, H. J. Reprocessing and recycling of thermosetting polymers based on bond exchange reactions. RSC Adv. 2014, 4 (20), 10108-10117. (c) Imbernon, L.; Norvez, S.; Leibler, L. Stress Relaxation and SelfAdhesion of Rubbers with Exchangeable Links. Macromolecules 2016, 49 (6), 2172-2178.

(5) Denissen, W.; Winne, J. M.; Du Prez, F. E. Vitrimers: permanent organic networks with glass-like fluidity. Chemical Science 2016, 7 (1), $30-38$.

(6) (a) Denissen, W.; Rivero, G.; Nicolay, R.; Leibler, L.; Winne, J. M.; Du Prez, F. E. Vinylogous Urethane Vitrimers. Adv. Funct. Mater. 2015, 25 (16), 2451-2457. (b) Denissen, W.; Droesbeke, M.; Nicolaÿ, R.; Leibler, L.; Winne, J. M.; Du Prez, F. E. Chemical control of the viscoelastic properties of vinylogous urethane vitrimers. Nat. Commun. 2017, 8, 14857.

(7) Fortman, D. J.; Brutman, J. P.; Cramer, C. J.; Hillmyer, M. A.; Dichtel, W. R. Mechanically Activated, Catalyst-Free Polyhydroxyurethane Vitrimers. J. Am. Chem. Soc. 2015, 137 (44), 14019-14022.

(8) Zheng, P.; McCarthy, T. J. A Surprise from 1954: Siloxane Equilibration Is a Simple, Robust, and Obvious Polymer Self-Healing Mechanism. J. Am. Chem. Soc. 2012, 134 (4), 2024-2027.

(9) (a) Canadell, J.; Goossens, H.; Klumperman, B. Self-Healing Materials Based on Disulfide Links. Macromolecules 2011, 44 (8), 2536-2541. (b) Lafont, U.; van Zeijl, H.; van der Zwaag, S. Influence of Cross-linkers on the Cohesive and Adhesive Self-Healing Ability of Polysulfide-Based Thermosets. ACS Appl. Mater. Interfaces 2012, 4 (11), 6280-6288. (c) Pepels, M.; Filot, I.; Klumperman, B.; Goossens, $\mathrm{H}$. Self-healing systems based on disulfide-thiol exchange reactions. Polym. Chem. 2013, 4 (18), 4955-4965. (d) Lei, Z. Q.; Xiang, H. P.; Yuan, Y. J.; Rong, M. Z.; Zhang, M. Q. Room-Temperature SelfHealable and Remoldable Cross-linked Polymer Based on the Dynamic Exchange of Disulfide Bonds. Chem. Mater. 2014, 26 (6), 2038-2046. (e) Martin, R.; Rekondo, A.; Ruiz de Luzuriaga, A.; Cabanero, G.; Grande, H. J.; Odriozola, I. The processability of a poly(urea-urethane) elastomer reversibly crosslinked with aromatic disulfide bridges. J. Mater. Chem. A 2014, 2 (16), 5710-5715. (f) Rekondo, A.; Martin, R.; Ruiz de Luzuriaga, A.; Cabanero, G.; Grande, H. J.; Odriozola, I. Catalyst-free room-temperature selfhealing elastomers based on aromatic disulfide metathesis. Mater. Horiz. 2014, 1 (2), 237-240. (g) Imbernon, L.; Oikonomou, E. K.; Norvez, S.; Leibler, L. Chemically crosslinked yet reprocessable epoxidized natural rubber via thermo-activated disulfide rearrangements. Polym. Chem. 2015, 6 (23), 4271-4278.

(10) (a) Taynton, P.; Yu, K.; Shoemaker, R. K.; Jin, Y.; Qi, H. J.; Zhang, W. Heat- or Water-Driven Malleability in a Highly Recyclable Covalent Network Polymer. Adv. Mater. 2014, 26 (23), 3938-3942. (b) Taynton, P.; Ni, H.; Zhu, C.; Yu, K.; Loob, S.; Jin, Y.; Qi, H. J.; Zhang, W. Repairable Woven Carbon Fiber Composites with Full Recyclability Enabled by Malleable Polyimine Networks. Adv. Mater. 2016, 28 (15), 2904-2909. (c) Taynton, P.; Zhu, C.; Loob, S.; Shoemaker, R.; Pritchard, J.; Jin, Y.; Zhang, W. Re-healable polyimine thermosets: polymer composition and moisture sensitivity. Polym. Chem. 2016, 7 (46), 7052-7056. (d) Whiteley, J. M.; Taynton, P.; Zhang, W.; Lee, S.-H. Ultra-thin Solid-State Li-Ion Electrolyte
Membrane Facilitated by a Self-Healing Polymer Matrix. Adv. Mater. 2015, 27 (43), 6922-6927. (e) Chao, A.; Negulescu, I.; Zhang, D. Dynamic Covalent Polymer Networks Based on Degenerative Imine Bond Exchange: Tuning the Malleability and Self-Healing Properties by Solvent. Macromolecules 2016, 49 (17), 6277-6284.

(11) Lu, Y.-X.; Tournilhac, F.; Leibler, L.; Guan, Z. Making Insoluble Polymer Networks Malleable via Olefin Metathesis. J. Am. Chem. Soc. 2012, 134 (20), 8424-8427.

(12) Röttger, M.; Domenech, T.; van der Weegen, R.; Breuillac, A.; Nicolaÿ, R.; Leibler, L. High-performance vitrimers from commodity thermoplastics through dioxaborolane metathesis. Science 2017, 356 (6333), 62-65.

(13) Obadia, M. M.; Mudraboyina, B. P.; Serghei, A.; Montarnal, D.; Drockenmuller, E. Reprocessing and Recycling of Highly CrossLinked Ion-Conducting Networks through Transalkylation Exchanges of C-N Bonds. J. Am. Chem. Soc. 2015, 137 (18), 6078-6083.

(14) (a) Mudraboyina, B. P.; Obadia, M. M.; Allaoua, I.; Sood, R.; Serghei, A.; Drockenmuller, E. 1,2,3-Triazolium-Based Poly(ionic liquid)s with Enhanced Ion Conducting Properties Obtained through a Click Chemistry Polyaddition Strategy. Chem. Mater. 2014, 26 (4), 1720-1726. (b) Zhang, P.; Li, M.; Yang, B.; Fang, Y.; Jiang, X.; Veith, G. M.; Sun, X.-G.; Dai, S. Polymerized Ionic Networks with High Charge Density: Quasi-Solid Electrolytes in Lithium-Metal Batteries. Adv. Mater. 2015, 27 (48), 8088-8094.

(15) Van Ooteghem, D.; Deveux, R.; Goethals, E. J. Study of the reaction of sulfides and sulfonium salts. J. Sulphur Chem. 1972, 8 (Number 1).

(16) (a) Hoyle, C. E.; Lee, T. Y.; Roper, T. Thiol-enes: Chemistry of the past with promise for the future. J. Polym. Sci., Part A: Polym. Chem. 2004, 42 (21), 5301-5338. (b) Hoyle, C. E.; Bowman, C. N. Thiol-Ene Click Chemistry. Angew. Chem., Int. Ed. 2010, 49 (9), 1540-1573. (c) van den Berg, O.; Dispinar, T.; Hommez, B.; Du Prez, F. E. Renewable sulfur-containing thermoplastics via $A B$-type thiol-ene polyaddition. Eur. Polym. J. 2013, 49 (4), 804-812. (d) Lyon, G. B.; Cox, L. M.; Goodrich, J. T.; Baranek, A. D.; Ding, Y.; Bowman, C. N. Remoldable Thiol-Ene Vitrimers for Photopatterning and Nanoimprint Lithography. Macromolecules 2016, 49 (23), 8905-8913. (e) Tibbits, A. C.; Yan, Y. S.; Kloxin, C. J. Covalent Incorporation of Ionic Liquid into Ion-Conductive Networks via Thiol-Ene Photopolymerization. Macromol. Rapid Commun. 2017, 38, 1700113.

(17) Van Damme, J.; van den Berg, O.; Brancart, J.; Vlaminck, L.; Huyck, C.; Van Assche, G.; Van Mele, B.; Du Prez, F. AnthraceneBased Thiol-Ene Networks with Thermo-Degradable and PhotoReversible Properties. Macromolecules 2017, 50 (5), 1930-1938.

(18) Logan, S. R. The kinetics of isotopic exchange reactions. J. Chem. Educ. 1990, 67 (5), 371.

(19) Kade, M. J.; Burke, D. J.; Hawker, C. J. The power of thiol-ene chemistry. J. Polym. Sci., Part A: Polym. Chem. 2010, 48 (4), 743-750.

(20) Dyre, J. C. The glass transition and elastic models of glassforming liquids. Rev. Mod. Phys. 2006, 78 (3), 953-972.

(21) For the calculation of the $T_{\mathrm{v}}$ the maxwell relation was used: visc $=G^{*}$ Tau*, with $G$ being the shear modulus. Using the tensile modulus $E^{\prime}$ measured by DMA, $G$ was calculated from the relation $G=E^{\prime}(1+$ $v)=E^{\prime} / 3$, with $v=0.5$, the Poisson's ratio for rubbers, and $E^{\prime}=1.1$ MPa. 